\title{
De los íberos al imperio romano. Evolución tecnológica del hierro
}

\author{
J. Simon", J. Tartera*, M. Marsal" y J. Auladell** \\ Resumen Desde el siglo VII a.C., en que se supone comenzó el desarrollo de la cultura íbera, hasta la \\ desaparición del imperio romano, siglo $\mathrm{V}$, se constata la evolución de la metalurgia del \\ hierro. En este trabajo se resumen los resultados de los estudios del material de hierro \\ hallado en excavaciones realizadas en la franja mediterránea y en el valle del Guadalquivir. \\ En los primeros estadios, las piezas se conformaban a partir de láminas de metal o de barras \\ de composición heterogénea, pero ya se practicaban la recristalización, el temple y la \\ cementación de forma rudimentaria mientras que en la época tardorromana la mayoría de \\ piezas se elaboraban a partir de barras macizas más purificadas, empleando los mismos \\ tratamientos térmicos y termoquímicos, posiblemente más perfeccionados.
}

Palabras clave Arqueometalurgia. Hierro.

\section{From the Ibers to the Roman empire. Technical evolution of the iron metallurgy}

\begin{abstract}
From the Iberian culture (VII century B.C.) to the end of the Roman Empire ( $5^{\text {th }}$ century) a constant evolution in the iron metallurgy was experienced. In this work we summarize the result of the study of ancient iron objects found in the excavations of the Mediterranean Coast and in Guadalquivir valley. Samples from Iberian and Roman deposits have been studied. In the first stages, the pieces were shaped from metal sheets or rods of heterogeneous composition, but techniques like recrystallization, hardening or carburising were also employed. At the end of the period, better refined iron was produced and more correct treatments were applied.
\end{abstract}

Keywords Archaeometallurgy. Iron.

\section{INTRODUCCIÓN}

Es comúnmente aceptado que la cultura íbera comienza en el siglo VII a.C. y la caída del imperio romano tiene lugar a inicios del siglo $\mathrm{V}^{[1]}$. Es de suponer que en tan dilatado espacio de tiempo la tecnología del hierro sufrió una considerable evolución. Al igual que otros investigadores, notablemente R. Pleiner ${ }^{[2]}$, que han expuesto las sucesivas etapas de la metalurgia del hierro en Grecia y su posterior difusión en la Europa occidental a medida que se extendía su cultura ${ }^{[3 \text { y } 4]}$, hemos pretendido dar una visión de esta evolución en una zona de la franja del litoral mediterráneo, a partir del estudio de los materiales férreos hallados en una serie de excavaciones y se ha comparado con material férreo del valle del Guadalquivir.

\section{MATERIALES Y PROCEDIMIENTO EXPERIMEN- TAL}

El estudio de restos arqueológicos de origen férreo debe hacer frente a dos imponderables: 1) La deficiente conservación de las piezas de hierro halladas en yacimientos debido a su degradación y 2) La imposibilidad de analizar las piezas que, por su buen estado de conservación, figuran en los museos.

Tampoco hemos de olvidar que, tal como ocurrió con la escritura, no debía tener los mismos conocimientos empíricos el forjador profesional de una gran urbe que el ocasional de un poblado rural que sólo trabajaba el hierro cuando éste era necesario para su uso ${ }^{[5]}$.

El material estudiado, íbero y romano, proviene de excavaciones realizadas en la franja de litoral 
mediterráneo que abarca desde la comarca del Maresme hasta la del Montsià y el material de la excavación del valle del Guadalquivir.

Los materiales de origen íbero examinados fueron: piezas de hierro y escorias del yacimiento de Les Guardies, Vendrell (Tarragona), datados entre los siglos IV, y I a.C. y del yacimiento íbero-romano El Camp de les Lloses, Tona (Barcelona), siglos II-I a.C.

De la época romana se han estudiado piezas y escorias del yacimiento de la Solana en la comarca del Garraf (siglos V-VI) y Castillo de Cornellá, en la provincia de Barcelona, (siglos II a.C.-inicios del siglo VI) y del Cortijo del Guerra, valle del Guadalquivir (época tardo-romana).

De los materiales estudiados por uno de los autores de este trabajo en su tesis doctoral, destacan: restos de un carro ${ }^{[6]}$, fragmentos de cuchillo, distintas piezas de hierro y armas de los siglos VII-II a.C. encontrados en yacimientos de las provincias de Barcelona y Tarragona. La figura 1 muestra la distribución geográfica de los yacimientos.

El estudio de las estructuras metálicas se ha realizado por microscopía óptica y por microscopía electrónica de barrido sobre muestras pulidas y atacadas $^{[7]}$. Los análisis se han efectuado por EDS.

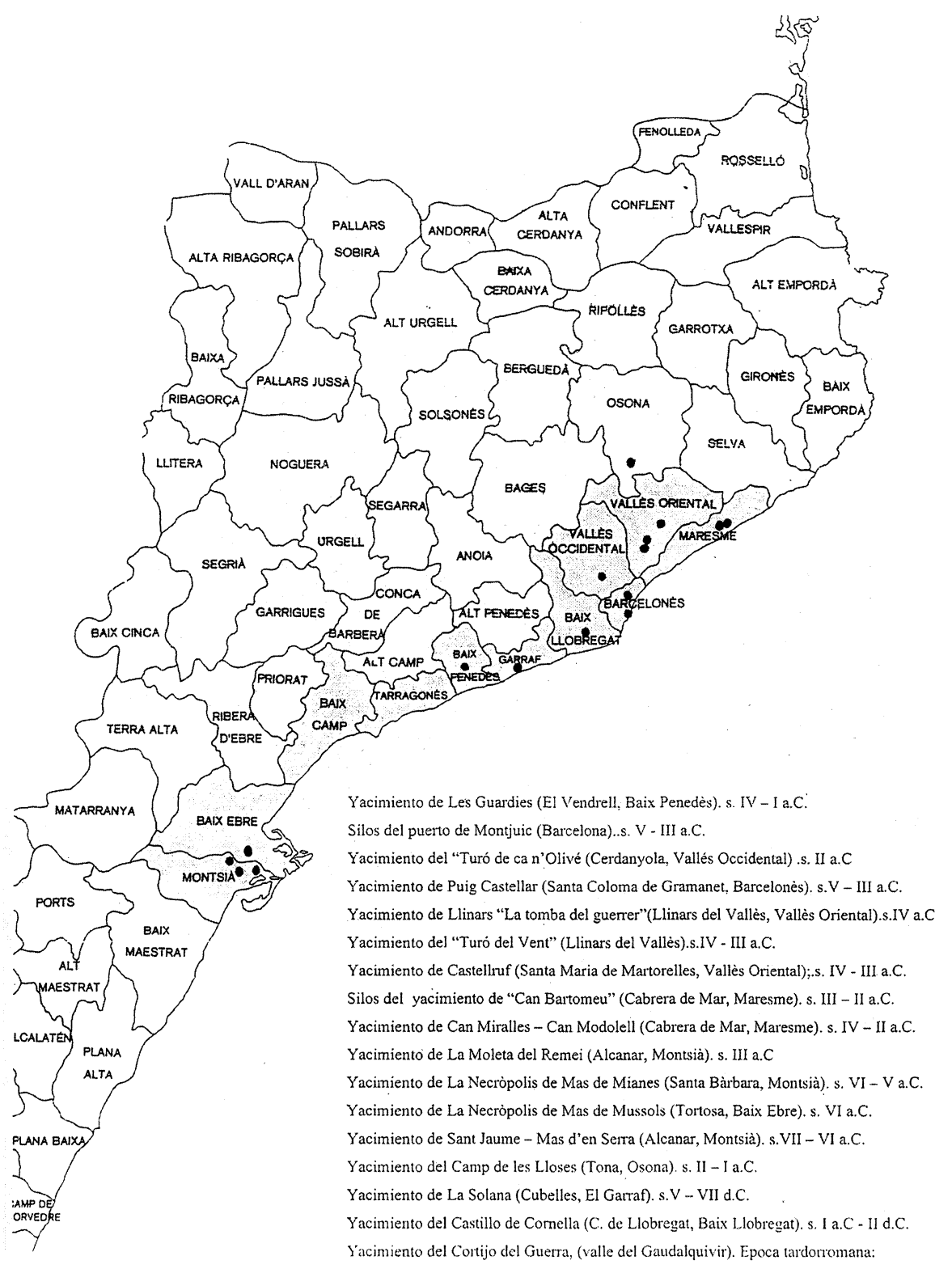

Figura 1. Distribución de las excavaciones arqueológicas con material férreo estudiado.

Figure 1. Geographic distribution of archeological excavation with the iron material studied. 


\section{RESULTADOS Y DISCUSIÓN}

La presencia de escorias y estructuras de combustión de Les Guardies permiten afirmar que el material metálico era autóctono. Todas las piezas fueron conformadas mediante unión por forja de láminas de hierro superpuestas en capas según dos técnicas distintas: A partir de una sola lámina por sucesivos plegamientos en capas, o bien por apilamiento de varias láminas ${ }^{[8]}$. De estas últimas cabe distinguir entre bloques forjados a partir de láminas de metal similar o las forjadas a partir de láminas de distinta composición. En las piezas estudiadas, lo habitual fue trabajar con láminas de aceros de bajo contenido en carbono. Prácticamente todas las piezas presentaban estructuras recristalizadas y en una aparecía una capa aparentemente cementada, difícil de confirmar debido al grosor de la capa de óxido superficial. Salvo en contados casos, la depuración del metal fue insuficiente, presentando restos escoriáceos atrapados en la matriz metálica. La unión entre láminas era defectuosa en muchas piezas.

Una de las piezas es un regatón (Fig. 2), en el cual la lámina interna es ferrítica, más dúctil y maleable, para adaptarla a la punta de la vara de madera, mientras que la capa externa, que debía ser más dura para que no se deformase, presenta una estructura ferrito-perlítica con algo de bainita (Fig. $3)$. Esto hace suponer que fue sometida a un temple suave. Las lanzas, se manufacturaron a partir de un apilamiento de láminas de acero, soldadas en caliente por martilleo. Una vez conformadas las piezas, se templaron para darle la dureza y resistencia requeridas a la punta de la lanza.

De las piezas encontradas en necrópolis, cabe destacar que se ha observado una gran diferencia en la técnica de conformación de las armas respecto a otros utensilios. En general, el material es

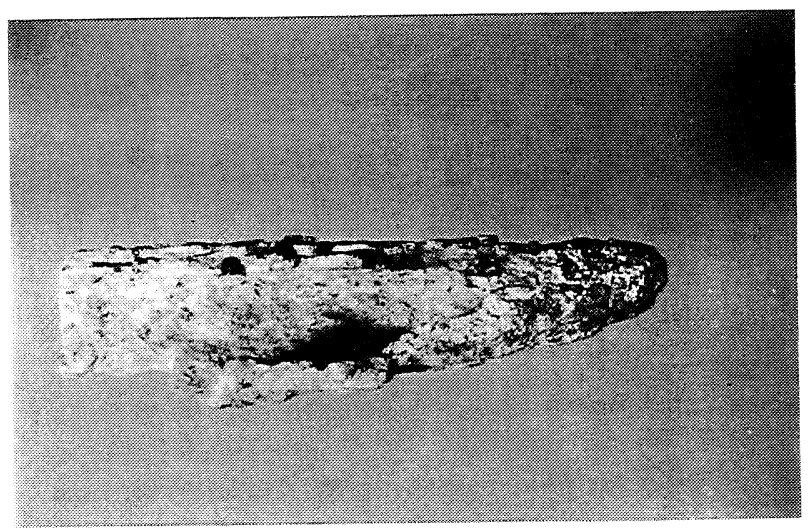

Figura 2. Regatón de hierro.

Figure 2. Iron shoulder.

Rev. Metal. Madrid Vol. Extr. (2005) 507-512

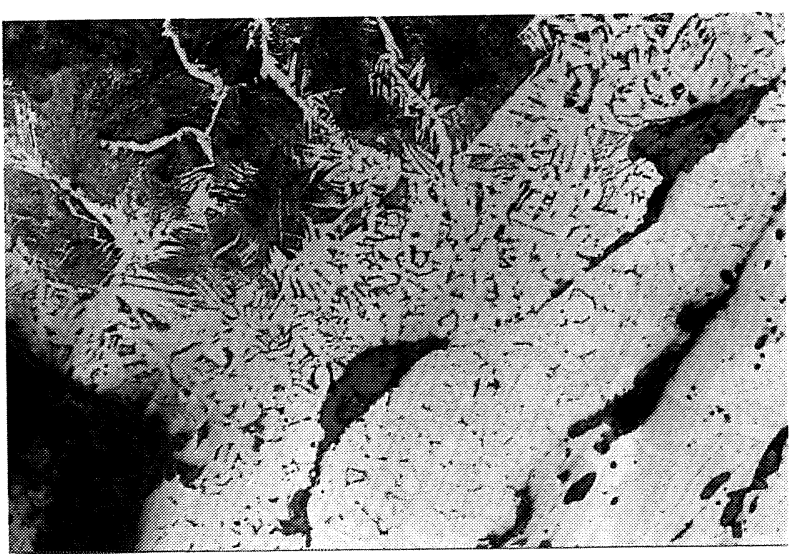

Figura 3. Regatón: lámina externa de microestructura ferrítico - perlítica y interna de microestructura ferríitica.

Figure 3. Shoulder: Outer layer with ferritic-perlitic microstructure and inner layer with ferritic microstructure.

de mayor calidad, exento de impurezas y mejor forjado, lo que demuestra los conocimientos empíricos de los trabajadores del metal que conformaron dichas armas. Desdichadamente, el ritual de incineración del difunto junto con su ajuar nos ha impedido precisar si dichas armas habían sufrido tratamientos térmicos y cuáles habían sido.

La mayoría de piezas del yacimiento del Camp de les Lloses estaban fabricadas mediante láminas apiladas de distinto porcentaje de carbono (Fig. 4), mientras que la fabricada a partir de una barra era de acero eutectoide (Fig. 5). Todas, estaban recristalizadas y, algunas, cementadas (Fig. 6).

El conjunto de material hallado en el yacimiento de la villa tardo-romana de La Solana demuestra que en ésta, se llevaba a cabo la cadena completa del trabajo del hierro, desde la reducción

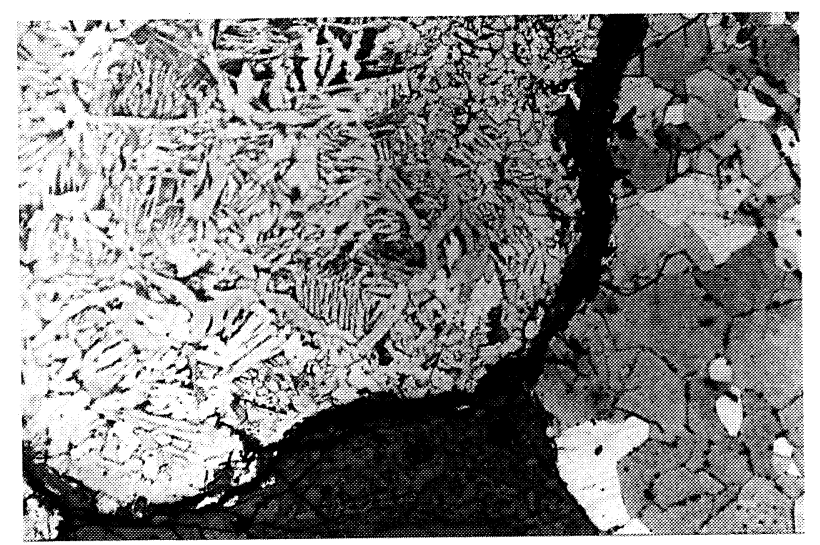

Figura 4. Pieza conformada a partir de láminas con distinto porcentaje de carbono.

Figure 4. Tool built up from layers with different carbon percentage. 


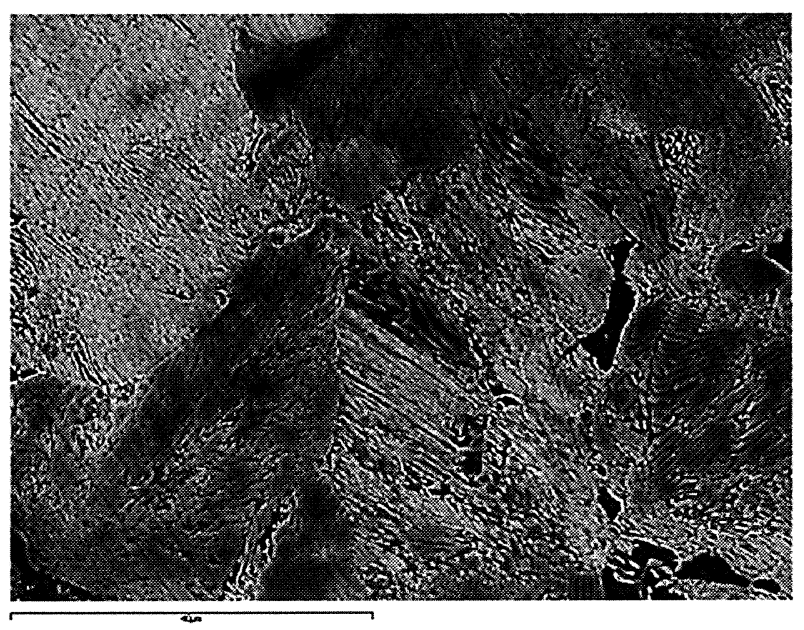

Figura 5. Pieza maciza de acero de microestructura eutectoide.

Figure 5. Bulk tool with a eutectoid steel microstructure.

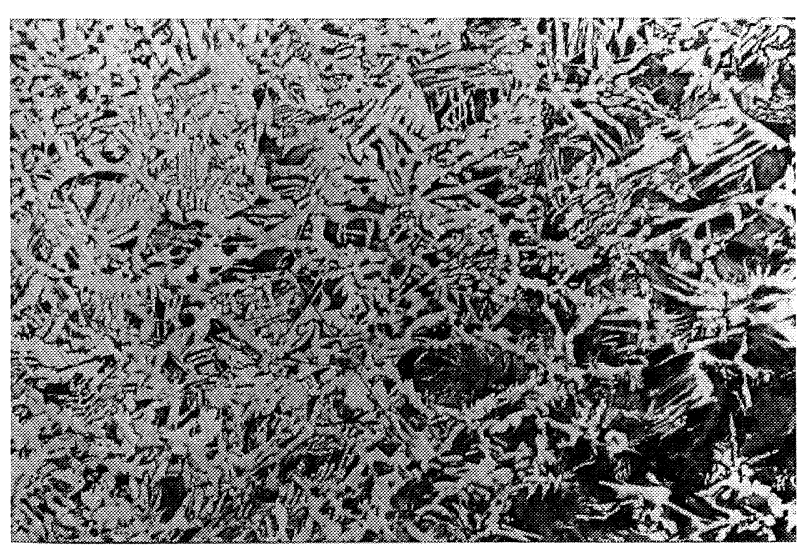

Figura 6. Borde cementado (inferior derecha) de una pieza de acero hipoeutectoide.

Figure 6. Carburized edge (lower left) of hipoeutectoid steel tool.

del mineral hasta la elaboración del objeto ${ }^{[9]}$. Las piezas estaban en distintas condiciones de conservación, desde útiles poco degradados hasta otros con alto grado de mineralización. Con aquellas que se pudo realizar un examen exhaustivo se concluyó que se utilizaron dos técnicas distintas: La conformación por plegamiento o apilamiento de láminas y la forja a partir de barra. El trabajo de depuración del hierro fue insuficiente, excepto en algunas armas. En todos los casos se procedió a una recristalización. La mayoría de piezas fueron conformadas a partir de hierro dulce y posteriormente cementadas. También se han encontrado fragmentos de utensilios confeccionados con láminas de metales distintos, hierro y cobre (Fig. 7) o bien de hierro y recubiertas con una capa de bronce. Te-

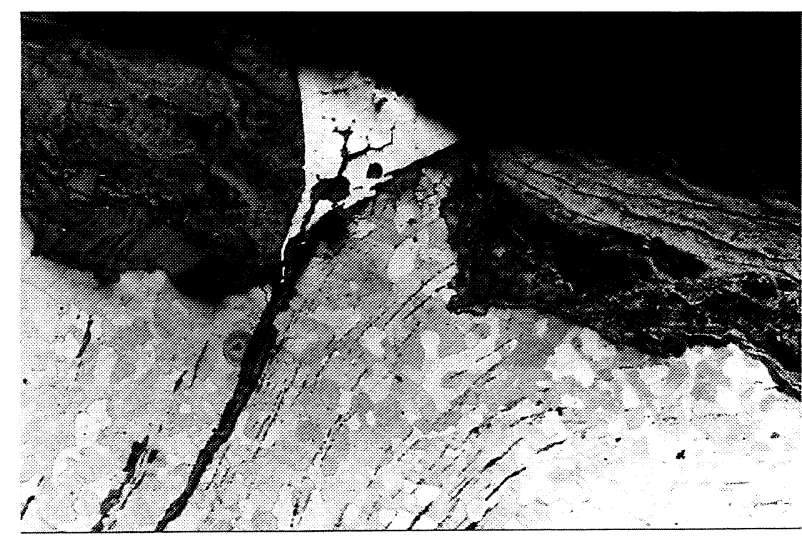

Figura 7. Penetración del cobre (blanco) entre dos láminas de hierro.

Figure 7. Copper penetration (white) between two iron layers.

niendo en cuenta que el material de La Solana estudiado abarca 150 años de historia, la desigualdad de calidad del producto manufacturado puede ser debida al distinto grado de eficiencia de artesanos que trabajaron en períodos de tiempo distintos. Sin embargo, las piezas están fabricadas con el material adecuado, lo que significa que tenían un buen conocimiento de éste y de las técnicas de trabajo que les permitía reservar lo mejor para aquellos objetos que más lo requerían.

En el material férreo del Castillo de Cornellà se constatan características tecnológicas similares al de La Solana. Las hojas de los cuchillos estaban conformadas a partir de una masa de metal que fue laminada y plegada por el lomo de la hoja (Fig. 8) ${ }^{[10]}$. El mismo procedimiento se empleó para los clavos: una lámina doblada sobre sí misma dejando un hueco central interno. En este hueco se encontraron restos de carbón vegetal. Como la pared interna del clavo estaba más carburada que el resto de la pieza es de suponer que el clavo se conformó doblando una lámina con polvo de carbón en su superficie que, a la temperatura de forja, actuó como cementante ${ }^{[11]}$.

Comparados con los objetos de la franja mediterránea, los del valle del Guadalquivir, pertenecientes a la época tardo-romana, son de formas más complejas, como puede observarse en una llave de templo (Fig. 9) o en una llanta de carro de combate. No obstante, ni el hierro utilizado estaba más depurado ni la conformación se había realizado con más pericia. El escaso número de piezas y la diversidad de las mismas no permiten sacar conclusiones que abarquen una interpretación conjunta. Sin embargo, algunas características son comunes en estos materiales: 


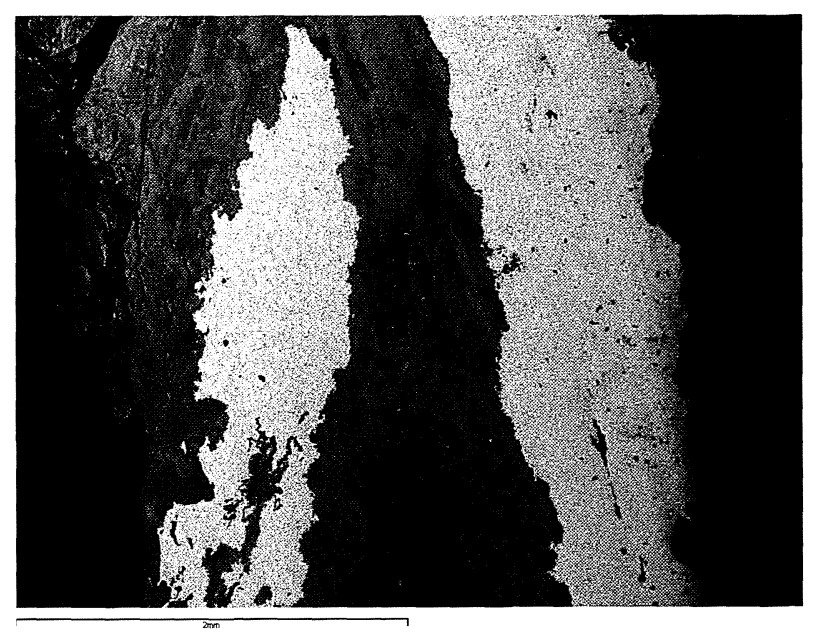

Figura 8. Porción del pliegue que conforma el cuchillo.La zona blanca es la metálica, la zona gris es la mineralizada.

Figure 8. Part of the folding that builds up the knife. The white area is metallic and the gray area is mineralized.

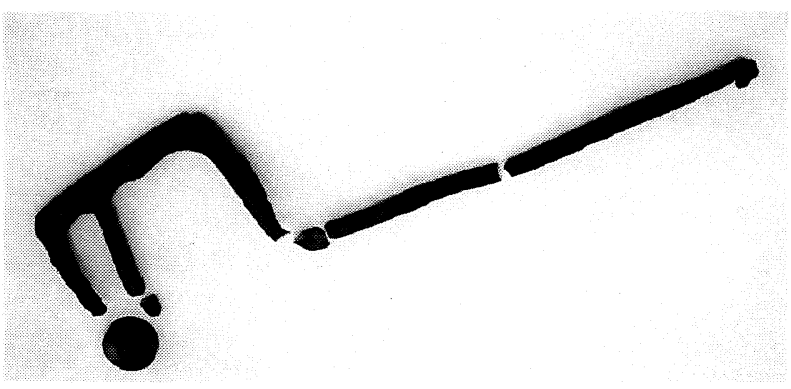

Figura 9. Llave de templo objeto de estudio.

Figure 9. Temple key that wos studied.

La mayor parte de los objetos habían sido forjados a partir de una barra de hierro dulce. Generalmente, las piezas estaban cementadas sólo por un lado, lo que hace suponer que la carburación se realizaba dejándolas reposar sobre las brasas de la forja, con lo que el carbono penetraba únicamente por la cara en contacto con el carbón. La gran cantidad de inclusiones no metálicas indica que se partía de material poco depurado que, los análisis lo prueban, provenía de la soldadura de masas pequeñas de distinta composición.

\section{CONCLUSIONES}

En los inicios de la cultura íbera (siglo VII a.C.) ya se realizaban tratamientos térmicos de recristalización, temple y cementación. Cabe la duda de que algunos de los objetos que presentaban dichos tratamientos no fueran de origen autóctono ${ }^{[12]}$. Las piezas se conformaban a partir de láminas de metal
De los íberos al imperio romano. Evolución tecnológica del hierro J. Simon, J. TARTERA, M. MARSAL y J. Auladell

apiladas o dobladas, pasándose posteriormente a la conformación de barras macizas. Al principio la composición del metal era muy heterogénea mientras que en la época tardo-romana se empleó hierro de composición más homogénea.

Se constata que los forjadores tenían los conocimientos empíricos de los tratamientos pero les faltaba la pericia y la experiencia del buen artesano. En general, las armas son de mejor calidad que otros tipos de piezas.

Se ha pretendido dar una visión general de la evolución de la tecnología del hierro desde el mundo íbero al romano. Con ello, apenas se ha añadido una pincelada a este cuadro que se está construyendo y que podrá completarse cuando se concluyan muchas de las excavaciones iniciadas y se tenga la seguridad de que han salido a la luz los restos más representativos de los pueblos íberos y romanos y cuando se permita el estudio metalúrgico de las piezas depositadas en los museos que, a cambio de la extracción de una muestra minúscula, permitiría dar una interpretación tanto arqueológica como metalúrgica del estado de la técnica en este período.

\section{Agradecimientos}

Los autores agradecen el material proporcionado por el Departamento de Prehistoria, Historia Antigua y Arqueología de la Universitat de Barcelona y a los museos que han prestado el material para la tesis de J. Auladell.

\section{REFERENCIAS}

[1] J. Auladel y J. Simón, Pyrenae 28 (1997).

[2] R.J. FORBES, Metallurgy in Antiquity: a notebook for archaeologist and technologist, Leiden, 1950.

[3] J. GÓmEZ, J. PÉREZ-SUÑE y J. Simón, La farga catalana en el marc de l'Arqueologia siderúrgica, Ed. Govern d'Andorra, 1995.

[4] R.H. Greaves y B. Wrighton, Metalografía. Microscopía Práctica, Ed. Urmo, Bilbao, 1966.

[5] G. Magnusson, Proc. Populonia/Piombino 1983, Strasbourg, 1988.

[6] P.L. Pelet, Helvetia Archeologica (1970).

[7] J. Pérez Suñe, V. Revilla, J. Gómez, J. Pou y J. Simón, The importance of ironmaking, Ed. G. Magnusson, Stockholm, 1995.

[8] J. Pérez Suñe, V. Revilla, J. Gómez, J. Simón, M. Marsal y F. Plana, Recherches sur l'économie du fer en Mediterraneé nord-occidentale, CNRC, Ed. Monique Mergoil, Montagnac, France, 1998. 
De los íberos al imperio romano. Evolución tecnológica del hierro J. Simon, J. Tartera, M. Marsal y J. Auladell

[9] R. PLEINER, Iron working in ancient Greece, Prague, 1969.

[10] R. PLEINER, Early iron Metallurgy in Europe, Ed. Wertine et Muhly, London, 1980.

[11] V. Revilla, J. Pérez SuÑe, J. GÓmeZ y J. Simón, Cota Zero 13 (1997).
[12] J. SimÓn, et altri CD-R. La Farga. Evolució de l'obtenció del ferro a Catalunya, Ed. CICIT, 2001.

[13] R.F. TyLECOTE, The early history of Metallurgy in Europe, London, 1987. 